\title{
Sistemas de aeração e densidades de estocagem na qualidade da água e produção de
}

\section{tilápia do Nilo}

\author{
Aeration systems and stocking densities on water quality and production of Nile tilapia \\ Sistemas de aireación y densidades de almacenamiento en calidad del agua y producción de tilapia \\ del Nilo
}

Recebido: 16/06/2021 | Revisado: 21/06/2021 | Aceito: 06/07/2021 | Publicado: 17/07/2021

\author{
Maria Luiza Rodrigues de Souza \\ ORCID: https://orcid.org/0000-0001-5643-0841 \\ Universidade Estadual de Maringá, Brasil \\ E-mail: mlrsouza@uem.br \\ Elenice Souza dos Reis Goes \\ ORCID: https://orcid.org/0000-0003-2437-4800 \\ Universidade Federal da Grande Dourados, Brasil \\ E-mail: elenicegoes@ufgd.edu.br \\ Sergio do Nascimento Kronka \\ ORCID: https://orcid.org/0000-0003-4102-8117 \\ Universidade Estadual Paulista, Brasil \\ E-mail: skronka@yahoo.com.br \\ Newton Castagnolli \\ Universidade Estadual Paulista, Brasil \\ In memorian
}

\begin{abstract}
Resumo
Objetivando-se avaliar a qualidade da água e o desempenho de tilápias em diferentes sistemas de aeração e densidades de estocagem, conduziu-se um experimento em delineamento inteiramente casualizado, num fatorial $3 \times 3$ (três sistemas de aeração: sem aeração; aerador de compressor radial; aerador tipo chafariz) e três densidades de estocagem: (3, 6 e 9 peixes $/ \mathrm{m}^{3}$ ). Conduziu-se o experimento por 252 dias, utilizando alevinos revertidos (peso $16,3 \mathrm{~g}$ ) e arraçoados com dietas contendo $29,5 \%$ de proteína bruta. Considerou-se o peixe como unidade experimental para peso médio corporal final (PCF), ganho de peso diário (GPMD) e o tanque, para produção de biomassa (PBI), conversão alimentar aparente (CAA) e variáveis limnológicas. Foram observadas diferenças para oxigênio, amônia e ortofosfato. As aerações e as densidades influenciaram nas médias de oxigênio na água. A amônia foi influenciada apenas pela densidade. A PBI (D1 $=1,29, \mathrm{D} 2=1,91$ e D3=2,44 kg/m ${ }^{3}$ ) aumentou com os acréscimos na densidade, porém, reduziu o PCF, GPMD. A CAA não foi diferente entre os tratamentos. O maior PCF $(512,9 \mathrm{~g})$ e GPMD $(1,83 \mathrm{~g} / \mathrm{dia})$ foi na densidade 3 peixes $/ \mathrm{m}^{3}$, enquanto que, a maior PBI foi observado na densidade de 9 peixes $/ \mathrm{m}^{3}\left(2,44 \mathrm{~kg} / \mathrm{m}^{3}\right)$. Não há necessidade de aeração na menor densidade; para a intermediária, o compressor radial mostrou-se mais eficiente, enquanto para 9 peixes $/ \mathrm{m}^{3}$, os sistemas de aeração tiveram efeitos semelhantes. Com a interação das duas variáveis (densidade e aeração), a densidade mais indicada é 6 peixes $/ \mathrm{m}^{3}$, com aeração por compressor radial.
\end{abstract}

Palavras-chave: Aeração; Desempenho; Oreochromis niloticus; Produção de biomassa.

\begin{abstract}
Aiming to evaluate the water quality and performance of tilapia in different aeration systems and stocking densities, an experiment was conducted in a completely randomized design, in a $3 \times 3$ factorial (three aeration systems: no aeration; radial compressor aerator; fountain aerator) and three stocking densities: $\left(3,6\right.$ and $\left.9 \mathrm{fish} / \mathrm{m}^{3}\right)$. The experiment was carried out for 252 days, using reversed fingerlings (weight $16.3 \mathrm{~g}$ ) and rationed with diets containing $29.5 \%$ crude protein. The fish were considered as the experimental unit for final mean body weight (PCF), daily weight gain (GPMD) and the tank for biomass production (PBI), apparent feed conversion (CAA) and limnological variables. Differences were observed for oxygen, ammonia and orthophosphate. Aerations and densities influenced the averages of oxygen in the water. Ammonia was influenced only by density. The PBI $(\mathrm{D} 1=1.29, \mathrm{D} 2=1.91$ and D3 $=2.44 \mathrm{~kg} / \mathrm{m} 3)$ increased with the increases in density, however, it reduced the PCF, GPMD. CAA was not different between treatments. The highest PCF (512.9 g) and GPMD (1.83 g/day) was at the density of $3 \mathrm{fish} / \mathrm{m} 3$, while the highest GNP was observed at the density of $9 \mathrm{fish} / \mathrm{m}^{3}\left(2.44 \mathrm{~kg} / \mathrm{m}^{3}\right)$. There is no need for aeration at the lowest density; for the intermediate, the radial compressor was more efficient, while for $9 \mathrm{fish} / \mathrm{m}^{3}$, the aeration systems had similar effects. With the interaction of the two variables (density and aeration), the most suitable density is $6 \mathrm{fish} / \mathrm{m}^{3}$, with aeration by radial compressor.
\end{abstract}

Keywords: Aeration; Performance; Oreochromis niloticus; Biomass production. 


\begin{abstract}
Resumen
Con el objetivo de evaluar la calidad del agua y el desempeño de la tilapia del Nilo en diferentes sistemas de aireación y densidades de población, se realizó un experimento en un diseño completamente al azar, en un factorial 3x3 (tres sistemas de aireación: sin aireación; aireador de compresor; aireador radial; aireador de fuente) y tres densidades de población: (3, 6 y 9 peces $/ \mathrm{m}^{3}$ ). El experimento se llevó a cabo durante 252 días, utilizando alevines invertidos (peso $16,3 \mathrm{~g}$ ) y alimentados con ración ( $29,5 \%$ de proteína cruda). Los peces fueron considerados como la unidad experimental para el peso corporal medio final (PCF), la ganancia de peso diaria (GPMD) y el tanque para la producción de biomasa (PBI), la conversión aparente de alimento (CAA) y las variables limnológicas. Se observaron diferencias para oxígeno, amoniaco y ortofosfato. Los sistemas de aireación y las densidades influyeron en los valores de oxígeno en el agua. El amoníaco fue influenciado solo por la densidad. El PBI (D1 $=1.29, \mathrm{D} 2=1.91$ y D3 $\left.=2.44 \mathrm{~kg} / \mathrm{m}^{3}\right)$ aumentó con los aumentos de densidad, sin embargo, redujo el PCF, GPMD. No hubo diferencia para CAA entre tratamientos. Se concluye que el PCF más alto $(512,9 \mathrm{~g})$ y GPMD $(1,83 \mathrm{~g} /$ día $)$ fue a la densidad de 3 peces / $\mathrm{m}^{3}$, mientras que el PBI más alto se observó a la densidad de 9 peces $/ \mathrm{m} 3\left(2,44 \mathrm{~kg} / \mathrm{m}^{3}\right)$. No hay necesidad de aireación a la densidad más baja; para el intermedio, el compresor radial resultó ser más eficiente, mientras que para 9 peces $/ \mathrm{m}^{3}$, los sistemas de aireación tuvieron efectos similares. Con la interacción de las dos variables (densidad y aireación), la densidad más adecuada es de 6 peces $/ \mathrm{m}^{3}$, con aireación por compresor radial.
\end{abstract}

Palabras clave: Aireación; Actuación; Oreochromis niloticus; Producción de biomasa.

\title{
1. Introdução
}

As tilápias representam o quarto grupo de peixes mais produzido no mundo (FAO, 2018), sendo a tilápia do Nilo (Oreochromis niloticus) a principal dentre elas, pois existem cerca de 77 espécies de tilápias descritas e distribuídas basicamente nos três gêneros: Tilapia, Sarotherodon e Oreochromis. Dentre as espécies descritas, quatro têm- se destacado na aquicultura mundial, graças as suas características: tilápia nilótica ou do Nilo (O. niloticus), tilápia de Moçambique (O. mossambicus), tilápia azul ou tilápia áurea (O. aureus) e a tilápia de Zanzibar (O. urolepis hornorum) (Oliveira et al., 2007). No Brasil, também a tilápia do Nilo é a espécie mais cultivada, com produção de 486.155 toneladas em 2020 (Peixe BR, 2021).

$\mathrm{O}$ aumento da produção de tilápia em todo o mundo deve ser atribuído, além do excelente sabor e boas características zootécnicas (Kubitza, 2000), tais como alto crescimento e fertilidade, relativa resistência à temperatura de até 18 a $20^{\circ} \mathrm{C}$, resistência a enfermidades e rusticidade (Segovia, 2000). É ainda uma espécie que se adapta a diferentes sistemas de manejo, incluindo os tanques-redes e sistemas com fluxo contínuo, como "raceways" e sistemas fechados, propiciando altas produtividades. Todavia, estes incrementos são limitados pelo oxigênio dissolvido, problema que pode ser evitado utilizando um eficiente sistema para aeração da água dos viveiros (Coldebella et al., 2021).

A aeração mecânica supre a necessidade de oxigênio exigida pelos peixes e nos processos biológicos dentro da coluna d'água. Dentre os parâmetros da água, o oxigênio dissolvido é o mais importante para o crescimento e sobrevivência dos peixes, sendo considerado o principal limitador da produtividade (Qayyum et al., 2005). Mas, além da incorporação de oxigênio dissolvido na água, a aeração também diminui a estratificação térmica e distribui uniformemente o oxigênio dissolvido na coluna d'água, podendo melhorar as condições de qualidade de água, por redução do acúmulo de matéria orgânica nos tanques com aeração (Oakes, 2011).

Assim, geralmente os baixos teores de oxigênio na água é o primeiro problema no desenvolvimento da aquicultura intensiva. Além do oxigênio dissolvido na água, o teor de amônia que é produzida pelo metabolismo dos animais, é outro fator limitante ao incremento da produtividade, principalmente nas produções intensivas.

Para resolver tais problemas, o uso dos aeradores é de extrema importância. Os mais utilizados na piscicultura são os tipos "paddle whels" ou aeradores de pás e os do tipo "vertical pump" ou aeradores tipo chafariz (Coldebella et al., 2020). Mas, segundo os autores, pela diversidade de tamanhos e formatos dos viveiros de piscicultura o posicionamento, assim como a combinação de uso destes modelos de aeradores simultaneamente podem não ser tão eficientes, devendo-se fazer um eficiente monitoramento nos parâmetros da água, além do desempenho dos peixes. Portanto, com o crescimento da piscicultura torna-se imperativo o aprimoramento de tecnologias, visando um aumento no rendimento de biomassa, através do aumento da 
produtividade, com peixes que atendam às exigências mercadológicas.

O objetivo deste trabalho foi investigar as alterações na qualidade da água e no desempenho de tilápias durante o cultivo com diferentes sistemas para aeração e densidades de estocagem.

\section{Metodologia}

Esta pesquisa foi caracterizada como experimental (Pereira et al., 2018). Conduziu-se um experimento durante 252 dias (abril/dezembro), conforme relatado em Souza et al. (1998). Utilizaram-se 9 tanques (4,50 m largura x 9,0 m comprimento x 1,0 m profundidade), que tinham fundo de terra e taludes com tijolos inclinados. Os tanques possuíam abastecimento individual com água de igual procedência e vazão média para todos os tanques de aproximadamente 20 litros/seg/ha.

\section{Animais, delineamento experimental e manejo}

Utilizaram-se 2119 alevinos (peso inicial médio de 16,3 g), que passaram pelo processo de reversão sexual para machos. Dos peixes estocados, $20 \%$ foram marcados na região dorsal (anterior à nadadeira dorsal), com três miçangas coloridas (cada cor correspondendo um número) (Carneiro, 1978), de forma a permitir a avaliação do ganho de peso individual dos peixes.

$\mathrm{O}$ experimento foi conduzido utilizando delineamento inteiramente casualizado, em esquema fatorial $3 \times 3$, contendo três sistemas de aeração ( $A_{1}=$ sem aeração; $A_{2}=$ compressor radial e $A_{3}=$ aerador tipo chafariz) e três densidades de estocagem $\left(D_{1}=\right.$ 3 peixes $/ \mathrm{m}^{3} ; \mathrm{D}_{2}=6$ peixes $/ \mathrm{m}^{3}$ e $\mathrm{D}_{3}=9$ peixes $/ \mathrm{m}^{3}$ ), conforme descrito em Souza et al. (1998).

Utilizou-se ração FRI-RIBE ${ }^{\circledR}$, (peletizada e extrusada), com 29,5\% de proteína bruta e 4182 kcal de energia bruta/kg. A ração peletizada era fornecida na proporção de 0,5 a $6 \%$ do peso dos peixes, em função do tamanho do peixe e temperatura da água; a ração extrusada variou de 1 a $3 \%$ do peso médio, conforme observações de procura de alimento pelos peixes e verificação da temperatura da água. A quantidade de ração fornecida era reajustada nas biometrias, quando se pesavam $20 \%$ dos peixes de cada viveiro. A frequência alimentar foi 4 vezes por dia $(8,11,14$ e 17h), exceto no período de junho a agosto, em que a temperatura da água oscilou de 17,1 a $21^{\circ} \mathrm{C}$ e aos domingos, quando o arraçoamento foi efetuado duas vezes por dia (11 e $16 \mathrm{~h} 30 \mathrm{~min}$ ).

\section{Monitoramento da qualidade da água}

Duas vezes por dia ( 8 e 17h) foi aferida a temperatura da água. A cada 15 dias, às $8 \mathrm{~h}$, foi monitorado a amônia, oxigênio dissolvido, clorofila a, nitrato, nitrito, fósforo e ortofosfato, alcalinidade total, condutividade elétrica e pH da água dos viveiros, assim como também estudado por Ferreira et al. (2020). As amostras foram coletadas sempre no mesmo ponto, utilizando uma garrafa coletora, na profundidade de $60 \mathrm{~cm}$. A transparência foi medida com auxílio do disco de Secchi. As metodologias utilizadas nas análises dos parâmetros foram baseadas em Koroleff (1976), Golterman et al. (1978) e Nush (1980).

\section{Sistemas de aeração}

Foram testados os aeradores do tipo compressor radial e tipo chafariz, conforme descrito anteriormente em Souza et al. (1998). O aerador de compressor radial foi baseado em um motor de $2 \mathrm{HP}$, que sorvia ar atmosférico por uma turbina, direcionando-o a uma linha de distribuição, conectadas a duas linhas de abastecimento, feitas em tubo PVC 3/4" e contendo válvula de regulagem da quantidade de ar inserida por viveiro. As mangueiras de abastecimento foram colocadas no sentido longitudinal do fundo do tanque, possuindo um difusor de ar e válvula de retenção, nas regiões centras e terminal do viveiro.

No aerador tipo chafariz, em cada viveiro a água foi captada a $60 \mathrm{~cm}$ de profundidade por um motobomba de $0,5 \mathrm{HP}$ (através de um tubo PVC 3/4" com um crivo na extremidade), que a direcionava para uma das laterais do tanque, de onde partiam dois tubos com 1,0 m de altura, contendo um difusor de ar por extremidade, para saída da água, trabalhando semelhante aos chafarizes. 
O acionamento das aerações foi controlado através de um temporizador, sendo inicialmente, de abril a outubro, a aeração acionada das 0 às 5 h e, de outubro a dezembro, das 23 às $6 \mathrm{~h}$.

\section{Determinação do desempenho produtivo}

Ao término do experimento, foi realizado a biometria de todos os peixes, para análise do peso corporal médio final (PCF) e de cada tanque experimental, coletados aleatoriamente 30 peixes marcados para análise do ganho de peso final (GPF). Para tanto, o peixe foi considerado a unidade experimental, para essas variáveis analisadas, enquanto, o tanque, foi considerado para a produção de biomassa $\left(\mathrm{kg} / \mathrm{m}^{3}\right)$, conversão alimentar aparente (CAA) e variáveis limnológicas.

\section{Análise estatística}

Os dados foram submetidos à análise de variância (ANOVA) e as médias comparadas pelo teste de Tukey a 5\% de significância, utilizando o software Statistical Analysis System (SAS, SAS Inst. Inc. Cary, NC, USA).

\section{Resultados e Discussão}

\section{Parâmetros de qualidade de água}

As médias de temperatura da água oscilaram entre 17,1 a $28,4^{\circ} \mathrm{C}$ (manhã) e de 20,1 a $33,1^{\circ} \mathrm{C}$ (tarde). Sabe-se que o melhor crescimento da tilápia do Nilo ocorre entre as temperaturas de 25 a $30^{\circ} \mathrm{C}$, sendo 26 a $28^{\circ} \mathrm{C}$ o ótimo de conforto térmico (Vinatea \& Veja, 1995). Durante a fase experimental a variação mensal das médias oscilou de $19,9^{\circ} \mathrm{C}$ (junho) a $29,6^{\circ} \mathrm{C}$ (dezembro), o que pode ter afetado o desempenho dos peixes principalmente no mês de junho.

Os valores de pH da água dos viveiros variaram de 6,51 a 6,76. Estes índices encontram-se dentro da amplitude de 6,5 a 9,0, considerada apropriada para a criação de peixes (Sample, 1994). Entretanto, conforme se pode verificar na Tabela 1, os valores de pH estiveram em próximos de 6,5, considerado o valor mínimo satisfatório para a criação.

Verificou-se que o sistema de aeração e aa densidade de estocagem dos peixes sobre a variação no pH da água (Tabela 2). Neste estudo, os valores de pH não mostraram um padrão definido de variação ao longo dos meses, estando conforme resultados apresentados por Takino \& Cipólli (1988), enquanto no experimento de Yoshida (1996), os valores de pH nos diferentes tratamentos oscilaram entre 6,6 e 7,5.

Não foi observada diferença $(\mathrm{P}>0,05)$ para a transparência, condutividade e clorofila a, indicando que tais variáveis não influenciaram nos resultados obtidos (Tabelas 1 e 2). Analisando-se os sistemas de aeração, observa-se uma menor transparência nos tanques com o sistema-chafariz, enquanto em relação às densidades de estocagem a menor transparência foi observada no tratamento com 9 peixes $/ \mathrm{m}^{3}$, provavelmente devido à maior movimentação no tanque e à maior quantidade de excreções que induziu à uma grande proliferação do plâncton, aumentando assim a turbidez da água. Todavia, os valores não diferiram significativamente entre si, apesar de a baixa transparência da água estar relacionada com baixas concentrações de oxigênio nos viveiros, especialmente no período noturno (Boyd, 1979). Neste experimento, observando-se os sistemas de aeração, o menor valor de transparência foi verificado no aerador tipo chafariz (Tabela 2), o qual, contrariando aquele autor, proporcionou maiores valores de oxigênio dissolvido. Em relação às densidades, a menor transparência foi verificada na densidade de 9 peixes $/ \mathrm{m}^{3}$, que apresentou a menor concentração de oxigênio dissolvido (Tabela 2), no que concorda com a citação de Boyd (1979).

Segundo Boyd (1979) e Milstein et al. (1995), as medidas de transparência da água dos viveiros de piscicutura estão inversamente relacionadas com a biomassa fitoplanctônica. Isto pode ser confirmado, analisando-se a Tabela 2, onde na densidade de estocagem, as maiores transparências foram observadas nos tanques que apresentaram os menores teores de 
clorofila a (Tabela 2). As transparências ideais medidas com o disco de Secchi, segundo Galli (1984), é entre 30 e $40 \mathrm{~cm}$, sendo estes valores diferentes dos obtidos no presente experimento.

As médias da condutividade elétrica durante o experimento foram de 28,46 a $35,07 \mu \mathrm{S} / \mathrm{cm}$ (viveiros) e $26,13 \mu \mathrm{S} / \mathrm{cm}$ (canal de abastecimento) (Tabela 1). Na Tabela 2, foram apresentados os valores médios de condutividade da água, para os sistemas de aeração e densidades estudadas. Takino \& Cipólli (1988), citam que a tilápia do Nilo apresentou melhor desempenho em tanques com condutividade entre 42 a $67 \mu \mathrm{S} / \mathrm{cm}$ e pior crescimento, quando a condutividade oscilou entre 108 a $116 \mu \mathrm{S} / \mathrm{cm}$.

Tabela 1 - Produção de biomassa, conversão alimentar aparente (CAA) e as médias dos parâmetros limnológicos nos viveiros.

\begin{tabular}{|c|c|c|c|c|c|c|c|c|c|c|}
\hline \multirow{2}{*}{$\frac{\text { Variáveis }}{\text { Densidade }}$} & \multirow{2}{*}{$\begin{array}{c}\text { Unidade } \\
\text { peixes } / \mathrm{m}^{3}\end{array}$} & \multicolumn{3}{|c|}{$\begin{array}{c}\text { CONTROLE - SEM } \\
\text { AERAÇÃO }\end{array}$} & \multicolumn{3}{|c|}{ COMPRESSOR RADIAL } & \multicolumn{3}{|c|}{ CHAFARIZ } \\
\hline & & 3 & 6 & 9 & 3 & 6 & 9 & 3 & 6 & 9 \\
\hline Produção biomassa & $\mathrm{kg} / \mathrm{m}^{3}$ & 1,40 & 1,71 & 2,19 & 1,35 & 2,17 & 2,78 & 1,12 & 1,85 & 2,35 \\
\hline CAA & - & 1,83 & 2,01 & 2,02 & 1,82 & 1,88 & 1,92 & 1,96 & 1,95 & 2,11 \\
\hline Oxigênio dissolvido ${ }^{1}$ & $\mathrm{mg} / \mathrm{L}$ & 2,69 & 2,32 & 1,79 & 3,67 & 2,41 & 2,11 & 3,77 & 3,33 & 3,07 \\
\hline Amônia ${ }^{1}$ & $\mu \mathrm{g} / \mathrm{L}$ & 183,59 & 243,65 & 291,51 & 95,84 & 267,47 & 369,87 & 155,16 & 278,04 & 295,04 \\
\hline Clorofila $\mathbf{a}^{\mathbf{1}}$ & $\mu \mathrm{g} / \mathrm{L}$ & 157,37 & 306,67 & 253,87 & 67,8 & 202,58 & 403,03 & 181,9 & 285,75 & 234,69 \\
\hline Transparência $^{1}$ & $\mathrm{Cm}$ & 88,42 & 72,63 & 61,84 & 102,37 & 64,74 & 52,37 & 58,95 & 58,16 & 59,21 \\
\hline Alcalinidade & $\mathrm{mg} / \mathrm{L}$ & 14,78 & 16,67 & 16,22 & 15,01 & 15,65 & 18,39 & 15,95 & 18,11 & 16,76 \\
\hline Condutividade & $\mu \mathrm{S} / \mathrm{cm}$ & 30,08 & 32,74 & 31,27 & 28,46 & 32,17 & 35,07 & 32,35 & 34,21 & 32,69 \\
\hline Nitrato & $\mu \mathrm{g} / \mathrm{L}$ & 175,63 & 167,56 & 170,48 & 174,37 & 213,91 & 206,32 & 148,23 & 214,45 & 163,87 \\
\hline Nitrito & $\mu \mathrm{g} / \mathrm{L}$ & 9,54 & 11,35 & 13,12 & 5,57 & 15,31 & 30,65 & 8,96 & 18,89 & 13,46 \\
\hline Fósforo & $\mu \mathrm{g} / \mathrm{L}$ & 1292,9 & 2137,3 & 2134,9 & 662,8 & 1520,7 & 3597,1 & 1315,2 & 2349,4 & 2143,1 \\
\hline Ortofosfato & $\mu \mathrm{g} / \mathrm{L}$ & 87,69 & 179,06 & 163,6 & 40,69 & 117,47 & 190,17 & 195,75 & 220,06 & 236,26 \\
\hline $\mathrm{PH}$ & & 6,54 & 6,62 & 6,61 & 6,51 & 6,57 & 6,55 & 6,66 & 6,76 & 6,57 \\
\hline Peixes com $400 \mathrm{~g}$ & $\%$ & 67,24 & 36,73 & 14,57 & 66,35 & 51,38 & 37,42 & 73,91 & 44,04 & 36,26 \\
\hline
\end{tabular}

${ }^{1}$ Resultados também apresentados em Souza et al. (1998).

Os valores de clorofila a ficaram entre 67,8 e 403,03 $\mu \mathrm{g} / \mathrm{L}$ (Tabela 1), com média de 35,76 $\mu \mathrm{g} / \mathrm{L}$ na água de abastecimento. Para densidades e aerações não houve diferença $(\mathrm{P}>0,05)$ apesar de se observar uma tendência crescente na concentração de clorofila a, conforme aumento da densidade (Tabela 2).

A alcalinidade total da água dos viveiros também não foi influenciada pelas aerações e densidades (Tabela 2). Os valores obtidos de alcalinidade contradizem os apresentados por Boyd (1990), que considera ideal para o sucesso do cultivo, entre 20 e $40 \mathrm{mg} / \mathrm{l}$.

As médias de oxigênio dissolvido e amônia observados quinzenalmente ao longo do experimento mantiveram-se dentro dos parâmetros adequados para esta espécie, oscilando de 1,79 - 3,77 mg/L. As menores médias foram observados nos tanques contendo maiores densidades (Tabela 1).

Para as aerações, houve efeito significativo $(\mathrm{P}<0,05)$ nos teores de oxigênio dissolvido e densidades de estocagem (Tabela 2). Para o sistema de aeração registrou-se em média, 3,39; 2,73 e 2,27 mg/L para os sistemas chafariz, compressor radial e sem aeração, respectivamente. Relativamente às densidades, foram observadas 3,38; 2,69 e 2,32 mg/L de oxigênio dissolvido para os tanques com 3, 6 e 9 peixes $/ \mathrm{m}^{3}$, respectivamente. Observou-se, portanto, um decréscimo no oxigênio dissolvido do tanque aerado pelo sistema-chafariz para o controle, e da menor para a maior densidade. Contudo, o valor obtido para o compressor radial não foi diferente do controle e sistema chafariz. Também, o teor de oxigênio na densidade de 6 peixes $/ \mathrm{m}^{3}$, não diferiu entre as demais densidades ( 3 e 9 peixes $/ \mathrm{m}^{3}$ ). Sabe-se que a tilápia do Nilo é capaz de sobreviver em água com teores de oxigênio menores que 1,0 mg/L (Carvalho Filho \& Scott, 1995), porém os valores acima de $3 \mathrm{mg} / \mathrm{L}$ devem ser preconizados, de forma a não inibir o crescimento (Luquet, 1991).

As Figuras 1a e 1b mostram as oscilações do oxigênio dissolvido na água, conforme as densidades, em cada aeração, bem como os sistemas de aeração em cada densidade, e a relação com o oxigênio na água de abastecimento, que variou entre 4,07 e $7,1 \mathrm{mg} / 1$. 
As médias de oxigênio dissolvido apresentaram um decréscimo, do início para o final do experimento, em todos os tratamentos, fato explicado pelo aumento da biomassa dos peixes, elevação da temperatura da água dos viveiros (Figuras 1a e 1b). Lawson (1995) cita que a taxa de consumo de oxigênio varia com a temperatura da água, tamanho do peixe, nível de atividade e tempo decorrido após a alimentação. As Figuras 1a e 1b, evidenciam que os valores de oxigênio são maiores em todos os tratamentos, na fase inicial, correspondendo ao menor tamanho dos peixes (em torno de $16 \mathrm{~g}$ ) e nos meses mais frios (maio a agosto) onde a temperatura da água mais baixa, reduz o metabolismo dos peixes, aumentando a capacidade de retenção de oxigênio pela água. Segundo D`Silva \& Maughan (1996), verificaram que somente o oxigênio dissolvido e a amônia, apresentaram diferença significativa $(\mathrm{P}<0,05)$ nas densidades de $1,3,6,9$ e 12 peixes $/ \mathrm{m}^{3}$, em cultivo de 112 dias de tilápia vermelha em canais de irrigação.

Verificou-se, na fase final do experimento, que nas primeiras horas do dia, principalmente nos viveiros com maior densidade os peixes, independente da aeração se aglomeravam nos locais de entrada de água, certamente em busca de oxigênio.

Figura 1 - Variação do oxigênio dissolvido na água de abastecimento e tanques, comparando-se: a) diferentes densidades de estocagem dentro de cada aeração; b) aerações dentro de cada densidade.
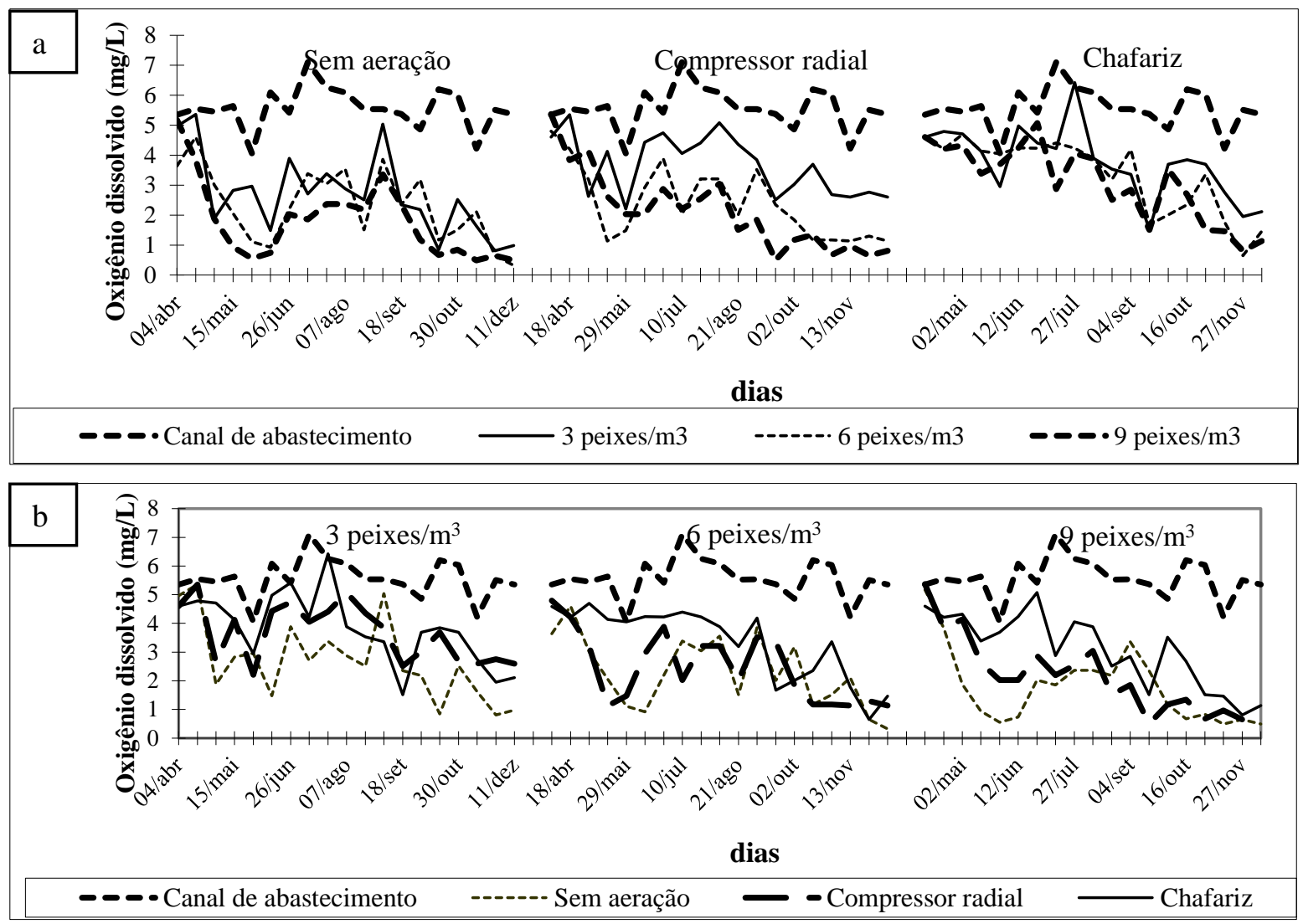

Fonte: Autores.

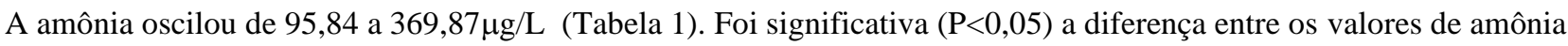
nas densidades de 3 e 9 peixes $/ \mathrm{m}^{3}$ (Tabela 2). Assim, quanto maior a densidade, maior era o teor de amônia nos tanques. A amônia não foi afetada pelas aerações (Tabela 2). Sabe-se que amônia e o oxigênio dissolvido dependem das densidades de estocagem, metabolismo dos peixes e o fluxo da água de abastecimento em pisciculturas (Degani et al., 1985). 
Research, Society and Development, v. 10, n. 8, e48010817238, 2021

(CC BY 4.0) | ISSN 2525-3409 | DOI: http://dx.doi.org/10.33448/rsd-v10i8.17238

Tabela 2 - Médias, teste F e coeficiente de variação dos parâmetros limnológicos nos viveiros.

\begin{tabular}{|c|c|c|c|c|c|c|c|c|c|c|c|}
\hline Variáveis & $\begin{array}{c}\text { Oxigênio } \\
(\mathrm{mg} / \mathrm{l})\end{array}$ & $\begin{array}{c}\text { Amônia } \\
(\mu \mathrm{g} / \mathrm{l})\end{array}$ & $\begin{array}{c}\text { Clorofila } \\
(\mu \mathrm{g} / \mathrm{l})\end{array}$ & $\begin{array}{c}\text { Nitrato } \\
(\mu \mathrm{g} / \mathrm{l})\end{array}$ & $\begin{array}{c}\text { Nitrito } \\
(\mu \mathrm{g} / \mathrm{l})\end{array}$ & $\begin{array}{c}\text { Fósforo } \\
(\mu \mathrm{g} / \mathrm{l})\end{array}$ & $\begin{array}{c}\text { Orto- } \\
\text { fosfato } \\
(\mu \mathrm{g} / \mathrm{l}) \\
\end{array}$ & $\begin{array}{l}\text { Álcali- } \\
\text { nidade } \\
(\mathrm{mg} / \mathrm{l})\end{array}$ & $\begin{array}{l}\text { Transpa- } \\
\text { rência } \\
(\mathrm{cm})\end{array}$ & $\begin{array}{c}\text { Conduti- } \\
\text { vidade } \\
(\mu \mathrm{S} / \mathrm{cm})\end{array}$ & $\mathrm{pH}$ \\
\hline \multicolumn{12}{|c|}{ SISTEMA DE AERAÇÃO (A) } \\
\hline$A_{1}-$ Sem aeração & $2,27 b^{(1)}$ & $239,58 \mathrm{a}$ & $239,30 \mathrm{a}$ & $171,22 \mathrm{a}$ & $11,34 \mathrm{a}$ & $1.855,03 \mathrm{a}$ & $143,45 \mathrm{ab}$ & $15,89 \mathrm{a}$ & $74,30 \mathrm{a}$ & $31,36 \mathrm{a}$ & $6,59 \mathrm{a}$ \\
\hline $\mathrm{A}_{2}$ - Compressor & $2,73 \mathrm{ab}$ & $244,39 \mathrm{a}$ & $234,11 \mathrm{a}$ & $198,20 \mathrm{a}$ & $17,18 \mathrm{a}$ & $1.926,87 \mathrm{a}$ & $116,11 \mathrm{~b}$ & $16,35 \mathrm{a}$ & 73,16 a & $31,90 \mathrm{a}$ & $6,54 \mathrm{a}$ \\
\hline $\mathrm{A}_{3}-$ Chafariz & $3,39 \mathrm{a}$ & $242,75 \mathrm{a}$ & $224,47 \mathrm{a}$ & $175,52 \mathrm{a}$ & $13,77 \mathrm{a}$ & $1.935,90 \mathrm{a}$ & $217,36 \mathrm{a}$ & $16,94 \mathrm{a}$ & $58,77 \mathrm{a}$ & $33,08 \mathrm{a}$ & $6,66 \mathrm{a}$ \\
\hline \multicolumn{12}{|c|}{ DENSIDADE DE ESTOCAGEM (D) } \\
\hline$D_{1}-3$ peixes $/ \mathrm{m}^{3}$ & $3,38 \mathrm{a}$ & $144,86 \mathrm{~b}$ & $135,69 \mathrm{a}$ & $166,08 \mathrm{a}$ & $8,02 \mathrm{a}$ & $1.090,30 \mathrm{a}$ & $108,04 \mathrm{a}$ & $15,25 \mathrm{a}$ & $83,25 \mathrm{a}$ & $30,30 \mathrm{a}$ & $6,57 \mathrm{a}$ \\
\hline$D_{2}-6$ peixes $/ \mathrm{m}^{3}$ & $2,69 a b$ & $263,05 \mathrm{ab}$ & $265,00 \mathrm{a}$ & $198,64 \mathrm{a}$ & $15,18 \mathrm{a}$ & $2.002,47 \mathrm{a}$ & $172,20 \mathrm{a}$ & $16,81 \mathrm{a}$ & $65,18 \mathrm{a}$ & $33,04 \mathrm{a}$ & $6,65 \mathrm{a}$ \\
\hline$D_{3}-9$ peixes $/ \mathrm{m}^{3}$ & $2,32 \mathrm{~b}$ & $318,81 \mathrm{a}$ & $297,20 \mathrm{a}$ & $180,22 \mathrm{a}$ & $19,08 \mathrm{a}$ & $2.625,03 \mathrm{a}$ & $196,68 \mathrm{a}$ & $17,12 \mathrm{a}$ & $57,81 \mathrm{a}$ & $33,01 \mathrm{a}$ & $6,58 \mathrm{a}$ \\
\hline \multicolumn{12}{|l|}{ Teste F } \\
\hline Aeração (A) & $12,24 *$ & $0,01 \mathrm{~ns}$ & $0,02 \mathrm{~ns}$ & $1,47 \mathrm{~ns}$ & $0,55 \mathrm{~ns}$ & $0,01 \mathrm{~ns}$ & $7,80 *$ & $0,71 \mathrm{~ns}$ & $1,31 \mathrm{~ns}$ & $0,74 \mathrm{~ns}$ & $3,69 \mathrm{~ns}$ \\
\hline Densidade (D) & $10,99 *$ & $11,12 *$ & $2,93 \mathrm{~ns}$ & $1,87 \mathrm{~ns}$ & $2,02 \mathrm{~ns}$ & $3,49 \mathrm{~ns}$ & $5,96 \mathrm{~ns}$ & $2,57 \mathrm{~ns}$ & $3,00 \mathrm{~ns}$ & $2,37 \mathrm{~ns}$ & $1,99 \mathrm{~ns}$ \\
\hline$\overline{\text { C.V. }(\%)}$ & 10,00 & 19,04 & 37,18 & 11,38 & 48,51 & 37,57 & 20,43 & 6,62 & 19,05 & 5,52 & 0,83 \\
\hline
\end{tabular}

${ }^{1}$ para cada fator, em cada coluna, médias seguidas de letras diferentes diferem entre si pelo teste de Tukey, ao nível de 5\% de significância. ns: não significativo (P>0,05) *significativo $(\mathrm{P}<0,05)$. Fonte: Autores. 
Os valores de nitrato variaram entre 148,23 $\mu \mathrm{g} / \mathrm{l}$ e 214,45 $\mu \mathrm{g} / \mathrm{l}$ (Tabela 1). Na Tabela 2, verifica-se ausência de diferença significativa $(\mathrm{P}>0,05)$ entre os teores de nitrato nos viveiros submetidos às diferentes aerações e densidades. No entanto, apesar ausência de significância, concentrações mais elevadas de nitrato foram observadas nos viveiros com aeração devido à oxidação do nitrito, confirmando a citação de Plemmons (1980), relatado por Thomforde \& Boyd (1991), que os valores de nitrato, em tanques aerados $(0,33 \mathrm{mg} / \mathrm{l})$, são superiores, comparados com não aerados $(0,03 \mathrm{mg} / \mathrm{l})$. Os níveis médios mantiveram-se dentro dos padrões admissíveis para a sobrevivência dos peixes, uma vez que o limite para a sobrevivência dos peixes e da vida aquática em geral é inferiror a 5 mg/l (Sipaúba-Tavares et al., 1994).

As médias de nitrito nos tanques oscilaram entre 5,57 a 30,65 $\mu \mathrm{g} / \mathrm{l}$ (Tabela 1). Não houve diferença para nitrito entre os tratamentos analisados. Os níveis de nitrito, medidos durante o experimento, mantiveram-se dentro dos padrões normais, pois, segundo Boyd (1981), o limite mínimo recomendável é de 1,0 mg/l. Os resultados estão próximos aos obtidos por Yoshida (1996), que observou variação de 0,0 a 32,20 $\mu \mathrm{g} / \mathrm{l}$ de nitrito, e ainda conforme Watenpaugh et al. (1985), há um acúmulo de nitrito nos viveiros, com acréscimo da densidade de estocagem, podendo reduzir a tolerância dos peixes. Ainda na Tabela 2, analisando os sistemas de aeração, observa-se um maior valor para o sistema-compressor radial, mesmo não sendo significativo entre as médias observadas nos diferentes tratamentos. Também pode ser evidenciado, que o tratamento sem aeração apresentou valor inferior aos aerados, confirmando os dados apresentados por Lai-Fa \& Boyd (1988), cujos valores foram 0,031 mg/l para tanques aerados e $0,028 \mathrm{mg} / \mathrm{l}$ para os controles (sem aeração).

As médias de fósforo total (662,8 a 3.597,1 $\mu \mathrm{g} / \mathrm{l})$ e ortofosfato (40,69 a 236,26 $\mu \mathrm{g} / \mathrm{l})$ nos diferentes tanques podem ser observadas na Tabela 1. Não se verificou diferença significativa em relação à densidade de estocagem (Tabela 2), porém podese observar que houve uma tendência de aumento na concentração de fósforo total e ortofosfato, à medida que aumentou a densidade de estocagem. Dentro dos sistemas de aeração os maiores valores médios de fósforo total e ortofosfato, foram observados nos sistema-chafariz, apesar, das médias de fósforo total não diferirem entre si, enquanto para ortofosfato foi significativo $(\mathrm{P}<0,05)$, sendo que o sistema-compressor radial apresentou o menor valor $(116,1 \mu \mathrm{g} / \mathrm{l})$ não diferindo apenas do tratamento sem aeração.

Os valores obtidos para o fósforo total, foram superiores aos resultados de diversos viveiros de piscicultura, entre $0,1 \mathrm{e}$ $87 \mu \mathrm{g} / \mathrm{l}$ (Sipaúba-Tavares, 1995). O mesmo não ocorreu com os dados apresentados por Yoshida (1996), que variaram de 0,0 a $25,87 \mathrm{mg} / \mathrm{l}$, sendo que o maior valor foi para o tanque sem renovação de água, comparado ao tanque sem renovação e com aeração e o tanque contendo renovação contínua da água. Quanto ao ortofosfato os valores foram inferiores a 1,7 mg/l, apresentado por Yoshida (1996). A liberação de compostos fosfatados pela decomposição da matéria orgânica, pode ter sido a causa das maiores concentrações de fósforo total e ortofosfato ocorrido neste experimento.

\section{Parâmetros de desempenho}

O peso final dos peixes, nos diferentes tratamentos pode ser observado na Tabela 3. Analisando-se as densidades de estocagem dentro do sistema de aeração, houve um decréscimo $(\mathrm{P}<0,05)$ no peso final, conforme aumento da densidade; no entanto, no aerador tipo chafariz as densidades 6 e 9 peixes $/ \mathrm{m}^{3}$, não diferiram. Resultados de D Silva e Maughan (1996) descrevem ausência de diferença $(\mathrm{P}>0,05)$ no peso nas densidades de 3, 6 e 9 peixes $/ \mathrm{m}^{3}$; todavia, naquele experimento os peixes foram mantidos por 112 dias em canais com intenso fluxo.

Outros autores relatam que o peso dos peixes diminui com o aumento na densidade de 1 peixes $/ \mathrm{m}^{2}$ para 3 peixes $/ \mathrm{m}^{2}$ (Diana et al., 1991), confirmando também os resultados da modelagem de crescimento proposto por Liu e Chang (1992), para densidades de 0,5 a 5 peixes $/ \mathrm{m}^{2}$. 
Tabela 3 - Médias de peso final (g) e ganho de peso total dos peixes marcados ( $\mathrm{g}$ ), teste $\mathrm{F}$ e coeficiente de variação (\%).

\begin{tabular}{|c|c|c|}
\hline Tratamentos & Peso final (g) & Ganho de peso diário $(\mathrm{g})$ \\
\hline $\begin{array}{l}\mathrm{A}_{1} \mathrm{D}_{1} \\
\mathrm{~A}_{1} \mathrm{D}_{2} \\
\mathrm{~A}_{1} \mathrm{D}_{3}\end{array}$ & $\begin{array}{l}492,63 \mathrm{Aa} \\
335,17 \mathrm{Bb} \\
278,82 \mathrm{Cb}\end{array}$ & $\begin{array}{l}1,83 \mathrm{Aa}^{(1)} \\
1,15 \mathrm{Bb} \\
0,97 \mathrm{Bb}\end{array}$ \\
\hline $\begin{array}{l}\mathrm{A}_{2} \mathrm{D}_{1} \\
\mathrm{~A}_{2} \mathrm{D}_{2} \\
\mathrm{~A}_{2} \mathrm{D}_{3}\end{array}$ & $\begin{array}{l}520,56 \mathrm{Aa} \\
411,66 \mathrm{Ba} \\
339,73 \mathrm{Ca}\end{array}$ & $\begin{array}{l}1,41 \mathrm{Ab} \\
1,45 \mathrm{Aa} \\
1,29 \mathrm{Aa}\end{array}$ \\
\hline $\begin{array}{l}\mathrm{A}_{3} \mathrm{D}_{1} \\
\mathrm{~A}_{3} \mathrm{D}_{2} \\
\mathrm{~A}_{3} \mathrm{D}_{3}\end{array}$ & $\begin{array}{l}525,61 \mathrm{Aa} \\
370,57 \mathrm{Bb} \\
352,14 \mathrm{Ba}\end{array}$ & $\begin{array}{l}1,62 \mathrm{Aab} \\
1,30 \mathrm{Bab} \\
1,19 \mathrm{Bab}\end{array}$ \\
\hline $\begin{array}{l}\text { Teste F } \\
\text { Interação (AxD) } \\
\text { C.V.(\%) }\end{array}$ & $\frac{2,61 *}{38,71}$ & $\frac{6,00 * *}{37,4}$ \\
\hline
\end{tabular}

(1) - Médias de D em cada nível de A (A em cada nível de D) seguidas de letra maiúscula (minúscula) diferentes denotam diferença pelo teste de Tukey. *significativo $(\mathrm{P}<0,05) * *$ significativo $(\mathrm{P}<0,01)$.

Analisando as aerações, não houve influência da aeração no peso final na menor densidade, enquanto as demais densidades, apresentaram diferença significativa $(\mathrm{P}<0,05)$. Em 6 peixes $/ \mathrm{m}^{3}$, o sistema-compressor radial proporcionou um peso final significativamente maior, quando comparado ao chafariz e sem aeração e estes dois não diferiram entre si; dentro da densidade 9 peixes $/ \mathrm{m}^{3}$, o compressor radial não diferiu significativamente do chafariz, porém ambos proporcionaram peso final maior que o controle.

A taxa de crescimento pode ter diminuído conforme aumento do número de peixes, devido, principalmente, aos baixos teores observados de oxigênio dissolvido. D`Silva e Maughan (1996), mencionam que a tilápia do Nilo, a exemplo de outras espécies, reduz o consumo alimentar em baixas qualidades da água, consequentemente afetando o seu crescimento. A tilápia do Nilo poderia ser cultivada em densidades acima de 10 peixes $/ \mathrm{m}^{2}$, entretanto, densidades elevadas tornam-se prejudiciais, devido aos metabólitos residuais na água (Suresh \& Lin, 1992).

Analisando a Tabela 3, as densidades, dentro de cada aeração, no tanque sem aeração e no chafariz, houve um decréscimo no ganho de peso diário, à medida que se aumentava a densidade de estocagem; entretanto, o desempenho dos peixes nos tanques com densidades de 6 e 9 peixes $/ \mathrm{m}^{3}$, não diferiram entre si ( $\left.\mathrm{P}>0,05\right)$. Já, nos tanques aerados com o sistema-compressor radial, não se observou diferença significativa entre as densidades. Comparando-se as aerações, em cada densidade, observa-se que em 6 e 9 peixes $/ \mathrm{m}^{3}$, o compressor radial proporcionou um maior ganho de peso diário aos peixes, apesar de não ter diferido significativamente do tipo chafariz, enquanto, na densidade 3 peixes $/ \mathrm{m}^{3}$, o menor ganho de peso foi para o compressor radial, o que se constitui aparentemente em um paradoxo de difícil explicação.

Os resultados observados são condizentes com os relatados por Colman et al. (1990), cuja taxa de ganho de peso diminui significativamente com acréscimo da densidade de estocagem, dentro de um mesmo regime alimentar nos diferentes tratamentos.

A produção de biomassa variou de 1,12 a $2,35 \mathrm{~kg} / \mathrm{m}^{3}$, cuja variação ocorreu em função do acréscimo da densidade de estocagem (Tabela 1). Ocorreu um aumentou $(\mathrm{P}<0,01)$ na produção de biomassa, à medida que aumentou a densidade de estocagem $\left(D_{1}=1,29 ; D_{2}=1,91\right.$ e $\left.D_{3}=2,44 \mathrm{~kg} / \mathrm{m}^{3}\right)$, enquanto não houve diferença $(P>0,05)$ entre os sistemas de aeração $\left(A_{1}=\right.$ 1,77; $\mathrm{A}_{2}=2,10$ e $\mathrm{A}_{3}=1,77 \mathrm{~kg} / \mathrm{m}^{3}$ ). Apesar de não ter ocorrido diferença significativa entre os sistemas de aeração, houve uma tendência a um melhor resultado para o sistema-compressor radial ( $\mathrm{A}_{2}$ ). Krom et al. (1989) citam que a qualidade da água é a principal limitação da produção de biomassa total de peixes em sistemas de cultivo. De acordo com Hollerman e Boyd (1980), a aeração proporciona um aumento na produção de peixes em tanques. Sendo assim, provavelmente o sistema-compressor radial proporcionou uma condição ambiental, mais favorável para o desenvolvimento dos peixes. 
Considerando-se a interação entre os dois fatores (densidade de estocagem e sistema de aeração), para a colheita da tilápia do Nilo, com 252 dias de cultivo, tanto para peso corporal (g), quanto para a produção de biomassa $\left(\mathrm{kg} / \mathrm{m}^{3}\right)$, a densidade mais indicada é a de 6 peixes $/ \mathrm{m}^{3}$, com aeração produzida pelo compressor radial, conforme se pode observar na Figura 2 .

Figura 2 - Peso final $(\mathrm{g})$ e produção de biomassa $\left(\mathrm{kg} / \mathrm{m}^{3}\right)$ dos peixes com 252 dias de cultivo.

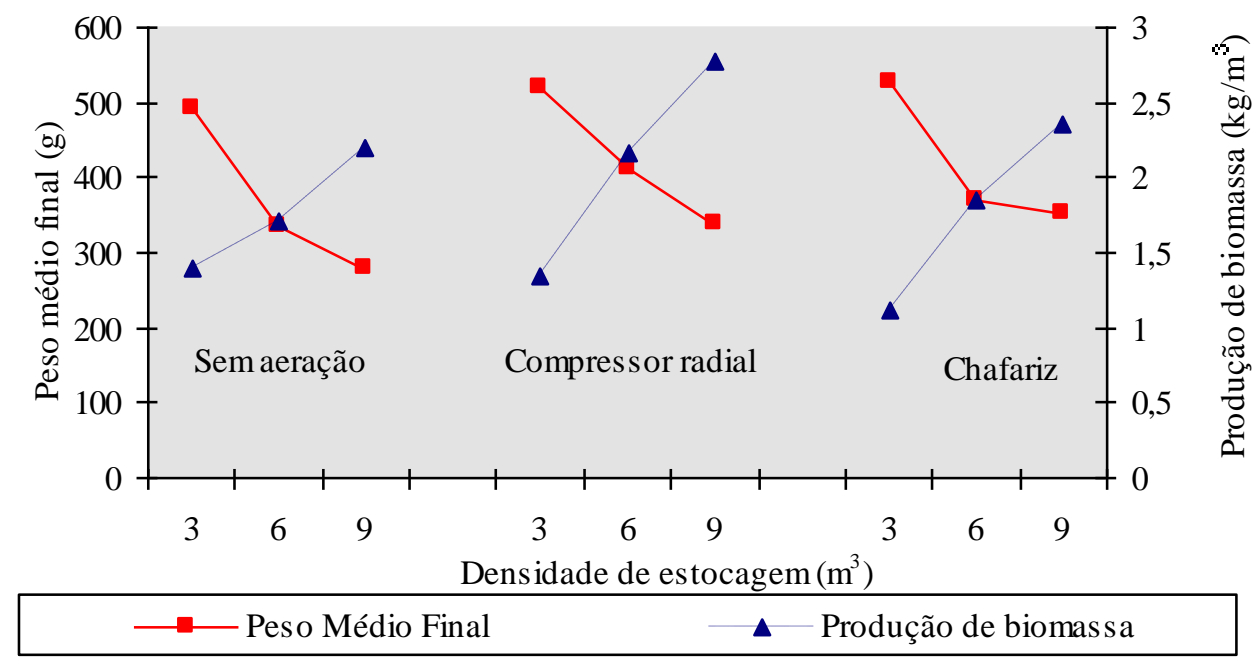

Fonte: Autores.

Quanto à conversão alimentar aparente, não houve diferença significativa entre os sistemas de aeração $\left(A_{1}=1,95 ; A_{2}=\right.$ 1,87 e $\left.A_{3}=2,00\right)$ e densidades de estocagem $\left(D_{1}=1,87 ; D_{2}=1,95\right.$ e $\left.D_{3}=2,02\right)$. Sendo assim, os resultados obtidos neste experimento são coerentes com os apresentados por Lovshin et al. (1990), no qual a CAA, apesar da baixa densidade (0,5 e 1 peixe $/ \mathrm{m}^{2}$ ), para tilápia do Nilo, não diferiu entre os tratamentos. No entanto, discordam dos relatados por Sarig \& Arieli (1980) e, cujos valores de CAA, diferiram entre os tratamentos, em diferentes densidades de estocagem. Todavia, observa-se na Tabela 1, que os melhores valores de CAA, foram obtidos na densidade de estocagem de 3 peixes $/ \mathrm{m}^{3}$ e os piores com 9 peixes $/ \mathrm{m}^{3}$, enquanto, comparando-se os sistemas de aeração, a melhor CAA foi obtida nos tanques com compressor radial $\left(A_{2}=1,87\right)$. Verificou-se, pois uma tendência de melhorar a CAA no sistema de aeração com compressor radial, na densidade de estocagem de 3 peixes $/ \mathrm{m}^{3}$. Segundo Tucker et al. (1979), a conversão alimentar aparente piora com o decréscimo do teor de oxigênio dissolvido na água, o que também é confirmado por Thomforde e Boyd (1991), que relatam, que a CAA foi significativamente melhor para bagre-do-canal, em tanques que receberam aeração no período noturno, comparado àqueles com aeração de emergência. Sarig e Arieli (1980) relatam que aumentando a densidade de estocagem, há um aumento na CAA, podendo ser evidenciadas tais afirmações nos resultados obtidos neste experimento, apesar dos resultados não terem sido significativos para esse parâmetro analisado. Em tambaquis (Colossoma macropomum), a taxa de CAA durante fase de recria foi de 1,8 (Silva et al., 2020).

\section{Porcentagem média de peixes terminados com peso corporal mínimo de $400 \mathrm{~g}$}

A porcentagem média de peixes terminados com peso corporal mínimo de $400 \mathrm{~g}$, variou de $28,71 \%$ a $69,21 \%$ (dados transformados através do arc sen), dentro da densidade de estocagem, enquanto, entre sistemas de aeração não houve diferença significativa $\left(A_{1}=38,36 \% ; A_{2}=51,68 \%\right.$ e $\left.A_{3}=51,78 \%\right)$. A porcentagem de peixes de porte superior a $400 \mathrm{~g}$, despescados na densidade de 3 peixes $/ \mathrm{m}^{3}\left(\mathrm{D}_{1}=69,21 \%\right)$ foi significativamente maior que nas densidades de $6\left(\mathrm{D}_{2}=43,99 \%\right)$ e de 9 peixes $/ \mathrm{m}^{3}\left(\mathrm{D}_{3}=28,71 \%\right)$, no entanto, estas duas densidades não diferiram entre si. 
Segundo Diana et al. (1991), ocorre um efeito da densidade sobre o peso do peixe. Liu e Chang (1992) consideram um bom resultado de despesca total, peixes que atingem peso médio de $200 \mathrm{~g}$ em densidade de estocagem de 2 peixes $/ \mathrm{m}^{2}$. O peso de abate depende da região onde a tilápia é cultivada, variando de 350 a 850 g.

\section{Conclusão}

Os níveis de oxigênio dissolvido e amônia mostraram-se dentro dos limites compatíveis de sobrevivência e desenvolvimento da tilápia do Nilo. Os demais parâmetros limnológicos parecem não ter influenciado no desempenho dos peixes. À medida que se aumentou a densidade de estocagem, observou-se aumento na produção de biomassa, porém, com redução do peso corporal e ganho de peso dos peixes. Não há necessidade de aeração para a menor densidade de estocagem (3 peixes $/ \mathrm{m}^{3}$ ); para a intermediária (6 peixes $\left./ \mathrm{m}^{3}\right)$, o compressor radial mostrou-se mais eficiente, embora para a maior densidade ( 9 peixes $\left./ \mathrm{m}^{3}\right)$, os sistemas de aeração tiveram efeitos semelhantes. $\mathrm{O}$ tratamento com menor densidade (3 peixes/ $\mathrm{m}^{3}$ ) proporcionou despesca de maior proporção de peixes com peso corporal médio acima de $400 \mathrm{~g}$, embora a aeração (Compressor radial ou chafariz) proporcionou maior produtividade. Todavia, analisando a interação das duas variáveis (densidade de estocagem e sistema de aeração), para a despesca da tilápia do Nilo, aos 252 dias de cultivo, tanto para peso corporal, ganho em peso, quanto produção de biomassa $\left(\mathrm{kg} / \mathrm{m}^{3}\right)$, o melhor resultado foi obtido com a densidade 6 peixes $/ \mathrm{m}^{3}$, com aeração produzida pelo compressor radial.

Novos estudos avaliando diferentes sistemas de aeração e outras densidades de estocagem, bem como períodos experimentais mais longos são importantes para aumentar os conhecimentos sobre a produção de tilápia em viveiros escavados.

\section{Referências}

Boyd, C. E. (1981). Water quality in warm water fish ponds. (2a ed.), Crafmaster Printers Inc. 359p.

Boyd, C. E. (1979). Determination of total ammonia nitrogen and chemical oxygen demand in fish culture systems.Transactions of the American Fisheries Society, 108, 314-319.

Boyd, C.E. (1990). Water quality in ponds for aquaculture. Birminghan Publishing, 483p.

Carneiro, D. J. (1978). Observações sobre a dinâmica de populações de peixes em uma represa. UNESP, 1978. 42p. Monografia (trabalho de graduação em Zootecnia) - Faculdade de Ciências Agrárias e Veterinárias do Campus de Jaboticabal.

Carvalho Filho, J.; Scott, P.C. (1995). Aspectos relevantes da biologia e do cultivo das tilápias. Panorama da Aqüicultura, 5(27), 8-13.

Colman, J. A., Edwards, P., Yomchinda, M., \& Pacharaprakiti, C. (1990). Extending the limits of fish production in manured static-water ponds. Aquaculture, 89(1), 27-41.

Coldebella, A., Gentelini, A. L., Mahl, I., Braun, N. J., Coldebella, P. F., Signor, A. A., \& Feiden, A. (2020). Perfiladores acusticos de corrente por efeito doppler (ADCP) como ferramenta de apoio para o posicionamento de aeradores na piscicultura de viveiros escavados. Brazilian Journal of Development, 6(9), 6458864600 .

D’Silva, A. M. \& Maughan, O. E. (1996). Optimum density of red tilapia Oreochromis niloticus x O. urolepis hornorum in a pulsed-flow culture system. Journal of the World Aquaculture Society, 27, 126-129.

Diana, J. S., Dettweiler, D. J., Lin, C. K. (1991). Effect of nile tilapia (Oreochromis niloticus) on the ecosystem of aquaculture ponds and its significance to the trophic cascade hypothesis. Canadian Journal of Fisheries Aquactic Sciences, 48, 183-190.

FAO. (2018). Organización De Las Naciones Unidas Para La Alimentación Y La Agricultura. El estado mundial de la pesca y la acuicultura. Departamento de Pesca y Acuicultura de la FAO. Roma, 2018.

Ferreira, L. K. S., dos Santos Cunha, D. A., Mesquita, S. L., Coelho, A. V., Junior, E. C. F., Bezerra, N. P. C., \& de Sousa, A. L. (2020). Indicadores de qualidade de água da criação do jurará em sistema intensivo (Kinosternon scorpioides Linnaeus, 1976). Research, Society and Development, 9(9), e36996543-e36996543. Galli, L.F. (1984). Introdução à piscicultura. Campinas: Fundação Cargill, 77p.

Golterman, H. L., Clymo, R. \& Ohsntad, M. A. M. (1978). Methods for physical and chemical analisys of freshwater. Boston: Blackwell Sci. Publ. 213p.

Hollerman, W. D. \& Boyd, C. E. (1980). Nightly aeration to increase production of Channel Catfish. Transactions of the American Fisheries Society, 109, 446452 .

Koroleff, F. (1976). Determination of nutrients. In: GRASSHOF, K. (Ed.) Methods of seawater analysis. Verlag Chemie Weinhein. $117-187$. 
Krom, M. D., Neori, A. \& Rijn, J. V. (1989). Importance of water flow rate in controlling water quality process in marine and freshwater fish ponds. The Israeli Journal of Aquaculture, 41, 23-33.

Kubitza, F. (2000). Tilápia: tecnologia e planejamento na produção comercial. 285 p.

Lai-Fa, Z. \& Boyd, C. E. (1988). Nightly aeration to increase the efficiency of channel catfish production. The Progressive Fish-Culturist, 50, $237-242$.

Lawson, T. B. (1995). Fundamentals of aquacultural engineering. Chapman \& Hall. 355p.

Liu, K. M. \& Chang, W. Y. B. (1992). Bioenergetic modelling of effects of fertilization, stocking density, and spawning on growth of the Nile tilapia, Oreochromis niloticus (L.). Aquaculture and Fisheries Management, 23, 291-301.

Lovshin, L. L., Tave, D. \& Lieutaud, A. O. (1990). Growth and yield of mixed-sex, young-of-the-year Oreochromis niloticus raised at two densities in earthen ponds in Alabama, U.S.A. Aquaculture, 89, 21-26.

Luquet, P. (1991). Tilapia, Oreochromis spp. In: Wilson, R.P. (Ed.). Handbook of nutrient requirements of finfish. London: CRC Press, p.169-179.

Milstein, A., Alkon, A., Karplus, I., Kochba, M., \& Avnimelech, Y. (1995). Combined effects of fertilization rate, manuring and feed pellet application on fish performance and water quality in polyculture ponds. Aquaculture research, 26(1), 55-65.

Oakes, P. L. (2011). Aeration of Ponds Used in Aquaculture. Agricultural Engineering, United States Department of Agriculture, Natural Resources Conservation Service-NRSC,Technical Note, No. AEN-3, 2011.

Oliveira, E. G.; Santos, F. J. S.; Pereira, A. M. L.; \& Lima, C. B. (2007). Produção de tilápia: Mercado, espécie, biologia e recria. Ministério da Agricultura, Pecuária e Abastecimento/ EMBRAPA. Circular técnica n.45.p.12.

Peixe BR. (2021). Associação Brasileira de Piscicultura. Anuário Peixe BR da Piscicultura 2021. PEIXE BR, 2021.

Pereira, S. A., Shitsuka, D. M., Parreira, F. J. \& Shitsuka, R. (2018). Metodologia da pesquisa científica. UFSM.

Qayyum, A., Ayub, M., \& Tabinda, A. B. (2005). Effect of aeration on water quality, fish growth and survival in aquaculture ponds. Pakistan Journal of Zoology, 37(4), 75 .

Sample, W. D. (1994). Water quality is essencial in holding fish in vats snd hauling tanks. Aquaculture Magazine, 3(20), 60-72.

Sarig, S. \& Arieli, Y. (1980). Growth capacity of tilapia in intensive culture. The Israeli Journal of Aquaculture, 32, 57-65.

Segovia, M. (2000). El cultivo de tilapia en sistemas cerrados en los Estados Unidos. Panorama Acuícola, 5(5), 26-29.

Silva, T. B. F., dos Santos Silva, R. R., do Nascimento Pinto, F. E., da Silva-Matos, R. R. S., Cordeiro, K. V., Pereira, A. M., \& Lopes, J. M. (2020). Criação de tambaqui associado à hidroponia em sistema de recirculação de água. Research, Society and Development, 9(9), e543997543-e543997543.

Sipaúba-Tavares, L. H. (1995). Limnologia aplicada à aqüicultura. FUNEP. 72p.

Sipaúba-Tavares, L. H. \& Colus, D. S. O. (1995). Estudo da variação nictemeral em um viveiro de piscicultura no período de seca. Revista Unimar, $17(2), 225-236$.

Souza, M. L. R., Castagnolli, N., \& Kronka, S. N. (1998). Influência das densidades de estocagem e sistemas de aeração sobre o peso e características de carcaça da tilápia do Nilo (Oreochromis niloticus Linnaeus, 1757). Acta Scientiarum. Animal Sciences, 20, $387-393$.

Suresh, A. V. \& Lin, C. K. (1992). Effect of stocking density on water quality and production of red tilapia in a recirculater water system. Aquacultural Engineering, 11, 1-22.

Takino, M. \& Cipólli, M. N. (1988). Caracterização limnológica em tanques de cultivo de tilápia, Oreochromis niloticus: Parâmetros físicos, químicos e clorofila a. Boletim do Instituto de Pesca, 15(2), 237-245.

Thomforde, H. W. \& Boyd, C. E. (1991). Effects of aeration on water quality and channel catfish production. The Israeli Journal of Aquaculture, 43(1): 3-26.

Tucker, L., Boyd, C. E. \& Mccoy, E. W. (1979). Effects of feeding rate on water quality, production of channel catfish, and economic returns. Transactions of the American Fisheries Society, 108, 389-396.

Vinatea, J. E. \& Vega, A. L. 1995. Piscicultura tropical: peces nativos y exóticos. Lima: Oficina General de Editorial. 338p.

Watenpaugh, D. E., Beitinger, T. L. \& Huey, D. W. (1985). Temperature tolerance of nitrite-exposed channel catfish. Transactions of the American Fisheries Society, 114, 274-278.

Yoshida, C. E. (1996). A dinâmica dos fatores físico-químicos em três tanques de piscicultura com renovação contínua, sem renovação da água e aeração artificial. Jaboticabal, 1996. 93p. Dissertação (Mestrado em Aquicultura), Faculdade de Ciências Agrárias e Veterinárias, Universidade Estadual Paulista. 